\title{
Mycoplasma genitalium as a sexually transmitted infection: implications for screening, testing, and treatment
}

\author{
J D C Ross, J S Jensen
}

Sex Transm Infect 2006;82:269-271. doi: 10.1136/sti.2005.017368

The evidence that Mycoplasma genitalium is a sexually transmitted pathogen is virtually incontrovertible based on both the concordance rates among partners and on DNA typing showing the same sequence type among partners in contrast to unrelated $M$ genitalium positive patients. The implications that this has for the screening, testing, and treatment of patients is less certain however. Which tests are the most sensitive and specific, what samples are most appropriate, who should be tested, what treatment is best and how should partners be managed?

See end of article for authors' affiliations

Correspondence to Professor J Ross, Whittall Street Clinic, Whittall Street, Birmingham B4 6DH, UK; jonathan.ross@ hobtpct.nhs.uk

Accepted for publication 23 March 2006
T he bacterium Mycoplasma genitalium is difficult to study. The organism is fastidious and culture is difficult and, even when successful, it takes several weeks or even months for each isolate to grow. Serology in its more sophisticated forms may have a role in epidemiological studies but is not of value in diagnosis. Hence, nucleic acid amplification tests (NAATs) are the only available diagnostic tools, but no commercially available test has been released for diagnostic purposes.

THE EVIDENCE FOR M GENITALIUM AS A PATHOGEN USING ANIMAL AND TISSUE MODELS

Studies in non-human primates have clearly demonstrated the pathogenicity of $M$ genitalium in both male and female animals. $M$ genitalium can be isolated from an infected animal and can be transferred to an uninfected animal and cause disease fulfilling one of Koch's postulates. ${ }^{1}$

In vitro studies demonstrate the potential for $M$ genitalium to attach to genital tract epithelial cells using a surface adhesin protein and then to enter the cells leading to the upregulation of cytokine genes with an associated inflammatory response. $^{2} M$ genitalium can also attach to spermatozoa giving a potential mechanism for spread to the female upper genital tract.

\section{EVIDENCE FOR M GENITALIUM AS A PATHOGEN IN MEN}

Large numbers of papers on the role of $M$ genitalium in male non-gonococcal urethritis (NGU) have been published since 1993 following the initial polymerase chain reaction based studies. ${ }^{4-6}$ Although different criteria have been used to define patient and control groups, all the studies have uniformly shown a higher prevalence of $M$ genitalium in the NGU groups $\left(\right.$ reviewed by Jensen ${ }^{7}$ ). Moreover, $M$ genitalium appears to be detected with the highest prevalence in men with Chlamydia trachomatis negative NGU (NCNGU). Several studies have found that men with $M$ genitalium positive NGU have symptoms as least as often as have those with chlamydial NGU. ${ }^{8}$ " When urethritis has been graded according to the number of polymorphonuclear leucocytes (PMNLs) in the urethral smear, men with $M$ genitalium have had higher PMNL counts than men with $M$ genitalium negative NCNGU, indicating a significant inflammatory potential. Systematic studies linking $M$ genitalium to complications such as epididymitis and prostatitis are lacking although $M$ genitalium DNA has been found both in the urethra of men with epididymitis, ${ }^{10}$ and in prostatic tissue of men with prostatitis. ${ }^{11}$

\section{EVIDENCE FOR M GENITALIUM AS A PATHOGEN IN WOMEN}

In women, $M$ genitalium can be detected in the genital tract and is found most commonly in those with genital tract symptoms or signs, or those who have an infected male partner. The presence of $M$ genitalium is associated with cervicitis and urethritis in women, ${ }^{12}{ }^{13}$ and the inoculation of $M$ genitalium in non-human primates leads to both lower genital tract disease and salpingitis. ${ }^{14} M$ genitalium can be detected in the endometrium of women with pelvic inflammatory disease ${ }^{15}$ and, on a single occasion, has been found in the fallopian tube. ${ }^{16}$ In addition, serological studies suggest a strong association between past infection with $M$ genitalium and tubal factor infertility. ${ }^{17}$

It therefore seems very likely that $M$ genitalium is a sexually transmitted pathogen in women ${ }^{9}$ and responsible for at least some cases of urethritis, cervicitis, and pelvic inflammatory disease.

\section{HOW TO DIAGNOSE M GENITALIUM: CURRENT AVAILABILITY OF TESTING AND FUTURE PROSPECTS}

At present, NAATs are the only tools available for detection of $M$ genitalium. Because of a very low load of mycoplasmas in some patients, ${ }^{18}$ tests with a very low limit of detection are needed in order to achieve sufficient assay sensitivity. No approved commercial assays have been made available although promising results with kits for research use have been presented. ${ }^{19}$ In the years

Abbreviations: NAATs, nucleic acid amplification tests; NGU, non-gonococcal urethritis; PMNLs,

polymorphonuclear leucocytes; STI, sexually transmitted infections 
to come, approved assays will most likely become available, but until then it is imperative that laboratories actively engage in external quality assurance programmes using real clinical specimens before they offer NAATs on a routine basis. The optimal specimen type may vary depending on the sample preparation method used in the laboratory. In one large study male first void urine was found to detect more infections with $M$ genitalium, as well as with Chlamydia trachomatis, than urethral swabs although this might reflect the amount of specimen used in the sample preparation method. In women the use of more than one specimen may also improve diagnostic sensitivity-for example, supplementing a urine specimen with a cervical swab. ${ }^{20}$

\section{ANTIBIOTIC THERAPY FOR M GENITALIUM INFECTIONS}

A number of different antibiotics have been used to treat $M$ genitalium infections with varying degrees of success. Tetracyclines initially looked promising but more recent studies suggest that failure to fully eradicate the infection occurs in a high proportion of cases treated with these agents. Macrolides, in particular azithromycin, offer the best chance of cure with a $84 \%$ clearance in a recent randomised controlled trial performed in men with $M$ genitalium urethritis. ${ }^{21}$ The newer quinolones, such as moxifloxacin, also have good activity against $M$ genitalium in vitro (although ciprofloxacin and ofloxacin are less effective). ${ }^{22}$

Because $M$ genitalium grows very slowly a prolonged course of therapy may be required to eradicate it. In a preliminary open study from Scandinavia a trend towards improved outcome with longer duration of therapy was observedazithromycin $1 \mathrm{~g}$ immediately eradicated $85 \%(11 / 13)$ of the $M$ genitalium infections whereas a dose of $500 \mathrm{mg}$ on day $\mathrm{l}$ followed by $250 \mathrm{mg}$ daily for 4 days eradicated 95\% (19/20) of infections. ${ }^{23}$

\section{Recommendations}

With the current state of knowledge of $M$ genitalium what interim recommendations can be given about screening, testing, and management?

\section{Screening}

It is premature to start population screening for $M$ genitalium. To do so we need accurate information on the prevalence of infection and prospective data on the natural history of disease in infected individuals. Only with this information can the efficacy and cost effectiveness of screening be calculated for different populations. The proposed testing of urine samples for $M$ genitalium from the NATSAL study ${ }^{24}$ will provide essential prevalence information to help inform the role of screening in the future. The evidence linking $M$ genitalium to pelvic inflammatory disease is strong but largely circumstantial and we still lack natural history studies which demonstrate a temporal relation between infection and disease. At least one such study is ongoing in the United States and another is planned for the United Kingdom, which will help to quantitate the risk of pelvic infection associated with $M$ genitalium infection and define the role of screening.

Testing

Although a variety of "in-house" PCRs have been developed there is a clear and urgent need for an accurate, standardised, and quality assured test kit for $M$ genitalium. Assuming a test is available who should be tested for $M$ genitalium?

Testing men with symptomatic NGU is reasonable, in particular in those settings where empirical treatment with doxycycline is used. Two thirds of the $M$ genitalium infected patients with urethritis will have persistent infection and often experience recurrent symptoms after doxycycline therapy. The same argument could also be used to justify

\section{Key messages}

- Mycoplasma genitalium is now established as a sexually transmitted infection

- The role of screening for infection is currently unknown

- Testing and treatment of symptomatic individuals infected with M genitalium is recommended, but limited by the lack of suitable tests

- Current evidence supports the use of azithromycin as first line therapy for $M$ genitalium

testing patients presenting with complications such as epididymitis, prostatitis, and sexually acquired reactive arthritis.

Testing for $M$ genitalium in women presenting with genital tract symptoms, such as genital discharge, intermenstrual bleeding, or pelvic pain is justified because of the association between $M$ genitalium and cervicitis, endometritis, and clinical pelvic inflammatory disease. Further information on the natural history and prevalence of infection is needed before testing of asymptomatic women can be recommended.

\section{Treatment}

Specific treatment for $M$ genitalium is appropriate in symptomatic patients in whom the organism has been detected and current evidence suggests that first line therapy with a 5 day course of azithromycin would be most appropriate. Single doses of azithromycin may be less effective in men with urethritis and occasionally macrolide resistance has been encountered. Patients with treatment failure after azithromycin have been successfully treated with moxifloxacin $400 \mathrm{mg}$ daily for 10 days $^{25}$ but because of the risk of development of resistance this treatment should be considered second line.

The lack of prospective natural history data makes a firm recommendation to trace and treat all sexual contacts premature at present, but such an approach is reasonable for individual patients after appropriate discussion.

\section{Summary}

Based on the current evidence a recommendation to test patients with genital symptoms for $M$ genitalium is justified and treatment of those found to be infected should be with azithromycin. The scope for testing will, however, be limited until validated and, preferably, commercially available tests become accessible.

\section{Authors' affiliations}

J D C Ross, Whittall Street Clinic, Birmingham B4 6DH, UK

J S Jensen, Statens Serum Institut, Artillerivej 5, DK-2300 Copenhagen S, Denmark

Declaration of interests: JR has received consultancy fees from Bayer and Pfizer and is an associate editor of Sexually Transmitted Infections.

$J R$ and JJ jointly wrote and revised the manuscript.

\section{REFERENCES}

1 Taylor-Robinson D. Mycoplasma genitalium - an up-date. Int J STD AIDS 2002;13:145-51

2 Zhang S, Wear DJ, Lo S-C. Mycoplasmal infections alter gene expression in cultured human prostatic and cervical epithelial cells. FEMS Immunol Med Microbiol 2000;27:43-50.

3 Svenstrup HF, Fedder J, Abraham-Peskir J, et al. Mycoplasma genitalium attaches to human spermatozoa. Hum Reprod 2003;18:2103-9.

4 Jensen JS, Orsum R, Dohn B, et al. Mycoplasma genitalium: a cause of male urethritis? Genitourin Med 1993;69:265-9.

5 Taylor-Robinson D, Horner PJ. The role of Mycoplasma genitalium in nongonococcal urethritis. Sex Transm Infect 2001;77:229-31. 
6 Horner PJ, Gilroy CB, Thomas BJ, et al. Association of Mycoplasma genitalium with acute non-gonococcal urethritis. Lancet 1993;342:582-5.

7 Jensen JS. Mycoplasma genitalium: the aetiological agent of urethritis and other sexually transmitted diseases. J Eur Acad Dermatol Venereol 2004; 18:1-11.

8 Falk L, Fredlund H, Jensen JS. Symptomatic urethritis is more prevalent in men infected with Mycoplasma genitalium than with Chlamydia trachomatis. Sex Transm Infect 2004;80:289-93.

9 Anagrius C, Lore B, Jensen JS. Mycoplasma genitalium:prevalence, clinical significance and transmission. Sex Transm Infect 2005;81:458-62.

10 Eickhoff JH, Frimodt-Moller N, Walter S, et al. A double-blind, randomized, controlled multicentre study to compare the efficacy of ciprofloxacin with pivampicillin as oral therapy for epididymitis in men over 40 years of age. Br J Urol Int 1999;84:827-34.

11 Krieger JN, Riley DE, Roberts MC, et al. Prokaryotic DNA sequences in patients with chronic idiopathic prostatitis. J Clin Microbiol 1996;34:3120-8.

12 Manhart LE, Critchlow CW, Holmes KK, et al. Mucopurulent cervicitis and Mycoplasma genitalium. J Infect Dis 2003;187:650-7.

13 Falk L, Fredlund $H$, Jensen JS, et al. Signs and symptoms of urethritis and cervicitis among women with or without Mycoplasma genitalium or Chlamydia trachomatis infection. Sex Transm Infect 2005;81:73-8.

14 Taylor-Robinson D, Furr PM, Tully JG, et al. Animal models of Mycoplasma genitalium urogenital infection. Isr J Med Sci 1987;23:561-4.

15 Cohen CR, Manhart LE, Bukusi EA, et al. Association between Mycoplasma genitalium and acute endometritis. Lancet 2002;359:765-6.

16 Cohen CR, Mugo NR, Astete SG, et al. Detection of Mycoplasma genitalium in women with laparoscopically diagnosed acute salpingitis. Sex Transm Infect 2005;81:463-6.

17 Clausen HF, Fedder J, Drasbek M, et al. Serological investigation of Mycoplasma genitalium in infertile women. Hum Reprod 2001;16:1866-74.
18 Jensen JS, Bjornelius E, Dohn B, et al. Use of TaqMan 5' nuclease real-time PCR for quantitative detection of Mycoplasma genitalium DNA in males with and without urethritis who were attendees at a sexually transmitted disease clinic. J Clin Microbiol 2004;42:683-92.

19 Wroblewski JK, Manhart LE, Dickey KA, et al. Detection of Mycoplasma genitalium in vaginal, cervical and urine specimens by TMA and PCR. 16th Biennial Meeting of the International Society for Sexually Transmittted Diseases Research, Amsterdam, 2005.

20 Jensen JS, Bjornelius E, Dohn B, et al. Comparison of first void urine and urogenital swab specimens for detection of Mycoplasma genitalium and Chlamydia trachomatis by polymerase chain reaction in patients attending a sexually transmitted disease clinic. Sex Transm Dis 2004;31:499-507.

21 Mroczkowski TF, Mena L, Nsuami M, et al. A randomised comparison od azithromycin and doxycycline for the treatment of Mycoplasma genitalium positive urethritis in men. ISSTDR Conference: Abstract WP-108, 2005

22 Hamasuna R, Osada Y, Jensen JS. Antibiotic susceptibility testing of Mycoplasma genitalium by TaqMan 5' nuclease real-time PCR. Antimicrob Agents Chemother 2005;49:4993-8.

23 Biornelius E, Anagrius C, Bojs G, et al. Mycoplasma genitalium: when to test and treat. Present status in Scandinavia. 15th Biennial meeting of the International Society for Sexually Transmittted Diseases Research, Ottawa, 2003.

24 Johnson AM, Mercer CH, Erens B, et al. Sexual behaviour in Britain: partnerships, practices, and HIV risk behaviours. Lancet 2001;358:1835-42.

25 Bradshaw CS, Tabrizi SM, Read TR, et al. Mycoplasma genitalium, herspes simplex viruses and adenoviruses in non-gonococcal urethritis. 16th Biennial Meeting of the International Society for Sexually Transmittted Diseases Research, Amsterdam, 2005. 